\title{
Moon Shadow by Cosmic Rays under the Influence of Geomagnetic Field and Search for Antiprotons at Multi-TeV Energies
}

\author{
M. Amenomori ${ }^{\text {a }}$, S. Ayabe ${ }^{\mathrm{b}}$, X. J. Bi ${ }^{\mathrm{c}}$, D. Chen ${ }^{\mathrm{d}}$, S. W. Cui ${ }^{\mathrm{e}}$, \\ Danzengluobu $^{f}$, L. K. Ding ${ }^{c}$, X. H. Ding ${ }^{f}$, C. F. Feng ${ }^{g}$, \\ Zhaoyang Feng ${ }^{c}$, Z. Y. Feng ${ }^{h}$, X. Y. Gao ${ }^{\text {i }}$, Q. X. Geng ${ }^{\mathrm{i}}$,

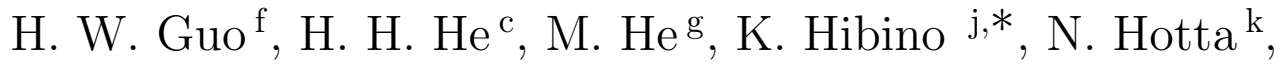 \\ Haibing $\mathrm{Hu}^{\mathrm{f}}$, H. B. Hu ${ }^{\mathrm{c}}$, J. Huang ${ }^{\ell}$, Q. Huang ${ }^{\text {h }}$, H. Y. Jia ${ }^{\text {h }}$,

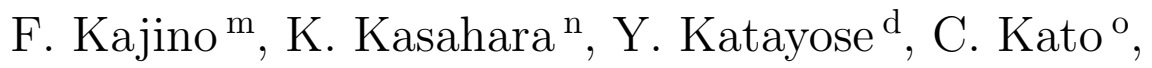 \\ K. Kawata ${ }^{\ell}$, Labaciren $^{f}$, G. M. Le ${ }^{\mathrm{p}}$, A. F. Li ${ }^{\mathrm{g}}$, J. Y. Li ${ }^{\mathrm{g}}$, \\ Y.-Q. Lou ${ }^{q}$, H. Lu ${ }^{c}$, S. L. Lu ${ }^{c}$, X. R. Meng ${ }^{f}$, K. Mizutani ${ }^{b, r}$, \\ J. Mu ${ }^{\mathrm{i}}$, K. Munakata $^{\circ}$, A. Nagai ${ }^{\mathrm{u}}, \mathrm{H}$. Nanjo ${ }^{\mathrm{a}}$, M. Nishizawa ${ }^{\mathrm{s}}$, \\ M. Ohnishi ${ }^{\ell}$, I. Ohta $^{t}$, H. Onuma ${ }^{\mathrm{b}}$, T. Ouchi ${ }^{\mathrm{j}}, \mathrm{S}$ Ozawa $^{\ell}$, \\ J. R. Ren ${ }^{\text {c }, ~ T . ~ S a i t o ~}{ }^{\text {v }}$, T. Y. Saito ${ }^{\ell}$, M. Sakata ${ }^{\mathrm{m}}$, T. K. Sako ${ }^{\ell}$, \\ T. Sasaki ${ }^{j}$, M. Shibata $^{d}$, A. Shiomi ${ }^{\ell}$, T. Shirai $^{\mathrm{j}}$, H. Sugimoto $^{\mathrm{w}}$, \\ M. Takita ${ }^{\ell}$, Y. H. Tan ${ }^{\mathrm{c}}$, N. Tateyama ${ }^{\mathrm{j}}$, S. Torii $^{\mathrm{r}}$, H. Tsuchiya ${ }^{\mathrm{x}}$, \\ S. Udo ${ }^{\ell}$, B. S. Wang ${ }^{\text {i, H. Wang }}{ }^{c}$, X. Wang ${ }^{\ell}$, Y. G. Wang ${ }^{g}$, \\ H. R. Wu ${ }^{\text {c }}$, L. Xue ${ }^{g}$, Y. Yamamoto ${ }^{\mathrm{m}}$, C. T. Yan ${ }^{\ell}$, X. C. Yang ${ }^{\mathrm{i}}$,

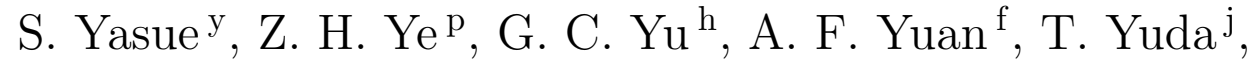 \\ H. M. Zhang ${ }^{\text {c }}$, J. L. Zhang ${ }^{c}$, N. J. Zhang ${ }^{g}$, X. Y. Zhang ${ }^{\mathrm{g}}$, \\ Y. Zhang ${ }^{\mathrm{c}}$, Yi Zhang ${ }^{\mathrm{c}}$, Zhaxisangzhu ${ }^{\mathrm{f}}$, X. X. Zhou ${ }^{\mathrm{h}}$ \\ (Tibet AS $\gamma$ Collaboration) \\ ${ }^{a}$ Department of Physics, Hirosaki University, Hirosaki 036-8561, Japan \\ ${ }^{\mathrm{b}}$ Department of Physics, Saitama University, Saitama 338-8570, Japan \\ ${ }^{\mathrm{c}}$ Key Laboratory of Particle Astrophysics, Institute of High Energy Physics, \\ Chinese Academy of Science, Beijing 100049, China \\ ${ }^{\mathrm{d}}$ Faculty of Engineering, Yokohama National University, Yokohama 240-8501, \\ Japan \\ ${ }^{\mathrm{e}}$ Department of Physics, Hebei Normal University, Shijiazhuang 050016, China \\ ${ }^{\mathrm{f}}$ Department of Mathematics and Physics, Tibet University, Lhasa 850000, China \\ g Department of Physics, Shandong University, Jinan 250100, China
}




\footnotetext{
${ }^{\mathrm{h}}$ Institute of Modern Physics, South West Jiaotong University, Chengdu 610031, China

${ }^{\mathrm{i}}$ Department of Physics, Yunnan University, Kunming 650091, China

${ }^{\mathrm{j}}$ Faculty of Engineering, Kanagawa University, Yokohama 221-8686, Japan

${ }^{\mathrm{k}}$ Faculty of Education, Utsunomiya University, Utsunomiya 321-8505, Japan

${ }^{\ell}$ Institute for Cosmic Ray Research, University of Tokyo, Kashiwa 277-8582, Japan

${ }^{\mathrm{m}}$ Department of Physics, Konan University, Kobe 658-8501, Japan

${ }^{\mathrm{n}}$ Faculty of Systems Engineering, Shibaura Institute of Technology, Saitama 330-8570, Japan

o Department of Physics, Shinshu University, Matsumoto 390-8621, Japan

${ }^{\mathrm{p}}$ Center of Space Science and Application Research, Chinese Academy of Sciences, Beijing 100080, C hina

${ }^{q}$ Physics Department and Tsinghua Center for Astrophysics, Tsinghua University, Beijing 100084, China

${ }^{\mathrm{r}}$ Advanced Research Institute for Science and Engineering, Waseda University, Tokyo 169-8555, Japan

${ }^{\mathrm{s}}$ National Institute for Informatics, Tokyo 101-8430, Japan

${ }^{\mathrm{t}}$ Tochigi Study Center, University of the Air, Utsunomiya 321-0943, Japan

u Advanced Media Network Center, Utsunomiya University, Utsunomiya 321-8585, Japan

`Tokyo Metropolitan College of Aeronautical Engineering, Tokyo 116-0003, Japan

${ }^{\mathrm{w}}$ Shonan Institute of Technology, Fujisawa 251-8511, Japan

${ }^{x}$ RIKEN, Wako 351-0198, Japan

${ }^{y}$ School of General Education, Shinshu University, Matsumoto 390-8621, Japan
}

\begin{abstract}
We have observed the shadowing of galactic cosmic ray flux in the direction of the moon, the so-called moon shadow, using the Tibet-III air shower array operating at Yangbajing (4300 m a.s.l.) in Tibet since 1999. Almost all cosmic rays are positively charged; for that reason, they are bent by the geomagnetic field, thereby shifting the moon shadow westward. The cosmic rays will also produce an additional shadow in the eastward direction of the moon if cosmic rays contain negatively charged particles, such as antiprotons, with some fraction. We selected $1.5 \times 10^{10}$ air shower events with energy beyond about $3 \mathrm{TeV}$ from the dataset observed by the Tibet-III air shower array and detected the moon shadow at $\sim 40 \sigma$ level. The center of the
\end{abstract}


moon was detected in the direction away from the apparent center of the moon by $0.23^{\circ}$ to the west. Based on these data and a full Monte Carlo simulation, we searched for the existence of the shadow produced by antiprotons at the multi$\mathrm{TeV}$ energy region. No evidence of the existence of antiprotons was found in this energy region. We obtained the $90 \%$ confidence level upper limit of the flux ratio of antiprotons to protons as $7 \%$ at multi-TeV energies.

Key words: Cosmic rays, Air shower, Anti matter, Observation and data analysis, Simulations

PACS: 96.50.sd, 94.20.wq, 25.43.+t, 95.30.-k, 95.75.-z, 98.80.-k

\section{Introduction}

Observation of antiproton abundance in cosmic ray flux raises the possibility of a baryon-symmetric universe and the propagation of cosmic rays in interstellar space. Recent measurements of antiproton flux, however, appear to be almost within the conventional cosmic ray physics in which antiprotons are produced as secondary particles of cosmic ray interactions with interstellar gas. For example, antiprotons are produced mainly by collisions of cosmic-ray protons with interstellar hydrogen gas as $p+p \longrightarrow \bar{p}+p+p+p$. Accelerator experiments measured the production rate $(\bar{p} / p)$ of antiprotons to protons to be on the order of $10^{-3}$ at energies greater than $10 \mathrm{GeV}$.

Recently, measurements of absolute flux of antiprotons below a few $\mathrm{GeV}$ were carried out using the magnet spectrometer equipped with a track detector to identify the charge and momentum of each incident particle [1,2,3,4]. Among these, the CAPRICE2 experiment extended the energy range of the spectrum up to about $50 \mathrm{GeV}$. It is obvious that accurate measurements of $\bar{p}$ flux are key to testing current propagation models of cosmic rays in the Galaxy. Of course, a $\bar{p}$ "excess" from the reliable propagation model may lead to discover possible sources of primary antiprotons such as dark matter annihilation and evaporation of primordial black holes. Strong et al. [5] made a detailed calculation of the antiproton flux, diffuse gamma rays and other cosmic ray fluxes and compared it with the recent BESS results available in the energy region below a few $\mathrm{GeV}$ [4]. They found that the conventional local cosmic ray measurements, simple energy dependence of the diffusion coefficient, and uniform cosmic ray source spectra through the Galaxy fail to reproduce simultaneously both the secondary to primary nuclei ratio and antiproton flux in this energy region.

\footnotetext{
* Corresponding author.

Email address: hibino@icrr.u-tokyo.ac.jp (K. Hibino ).
} 
The experiments mentioned above also present the flux ratio, $\bar{p} / p$, of antiprotons to protons in the cosmic rays. It seems that the observed results are within the scope of the prediction of the standard leaky box model [6]. Some results $[1,3]$, however, show a tendency that the $\bar{p} / p$ ratio increases with increasing primary energy, although the amount of data is insufficient.

In the simple leaky box model [7], the ratio of antiproton flux $f_{\bar{p}}\left(E_{\bar{p}}\right)$ to proton flux $f_{p}\left(E_{p}\right)$ is calculated as [8]

$$
\bar{p} / p \equiv f_{\bar{p}}\left(E_{\bar{p}}\right) / f_{p}\left(E_{p}\right) \propto \frac{\lambda_{e s c}\left(E_{p}\right)}{\lambda_{N}} Z_{N \bar{N}},
$$

where $\lambda_{\text {esc }} \equiv \rho \beta c \tau_{\text {esc }}$ ( $c$ is the light velocity) is the mean amount of matter (density $\rho$ ) traversed by a nucleon of velocity $\beta c, \tau_{e s c}$ is the mean time spent by the cosmic rays in the confinement space, and $\lambda_{N}$ is the mean free path of the nucleon in the interstellar matter. In addition, $Z_{N \bar{N}}$ is the production rate of antinucleons by nucleon - nucleon (hydrogen atom) interactions, which is almost constant when Feynman scaling holds, while weakly depending on the spectral index of cosmic rays. According to the HEAO-3 data on the $\mathrm{B} / \mathrm{C}$ ratio in the primary cosmic rays up to about $50 \mathrm{GeV} / \mathrm{n}, \lambda_{\text {esc }} \propto E^{-\delta}$ and $\delta \sim 0.6[9]$. Because the mean free path of the nucleon, $\lambda_{N}$, in this energy region is almost constant, the flux ratio of $\bar{p} / p$ should decrease with increasing primary energy. At high energies greater than $100 \mathrm{GeV}$, however, a value of $\delta \sim 0.3$ is expected because the cosmic ray anisotropy is less than $10^{-3}$ in the energy region below $100 \mathrm{TeV}$. Actually, $\delta$ should take a value of $1 / 3$ when the magnetic field in interstellar space has a Kolmogorov-type spectrum.

A closed galaxy model [10] predicts a considerable enhancement of secondary antiprotons. In this model, the cosmic ray flux observed locally in the solar system comprises young and old components. Cosmic rays in the young component show almost identical behavior to that discussed above, whereas the old component is contained in the galaxy halo. The old component of cosmic rays, however, consists only of protons because heavier nuclei in the old component are completely broken up during their long confinement time in the halo. Consequently, the value of $\lambda_{\text {esc }}$ for protons in the old component becomes very large. This model can engender a considerable enhancement of secondary antiprotons because of the existence of such old protons [11].

Until now, almost all measurements of antiprotons are limited in the energy region below a few $\mathrm{GeV}$ because of its extremely low flux and weight limit of the instrument on board the balloon. In this energy region, the interpretation of observed spectra is not free from uncertainties about the secondary antiproton production because of the nuclear threshold effect and because of uncertainties in the solar modulation effect. For those reasons, it is still difficult to distinguish which model is the best for explaining the antiproton 
spectrum. Considering the latest research, the possibility of the existence of antiprotons in the TeV energy region is slight, but we cannot exclude an exotic model such as an anti-galaxy production model yet (see the review paper by Stephen \& Golden [12]). Therefore, it will be important to measure $\bar{p} / p$ ratio in the TeV energy region. The results of such measurements might enable us to investigate the possible production of antiprotons from dark matter at high energies [13].

Urban et al. [14] first discussed the possibility of observing antiprotons using the moon shadow; the first observation was done by the Tibet-I experiment [15] by observing the sun shadow, giving an upper limit of the $\bar{p} / p$ ratio at 10 $\mathrm{TeV}[16]$. The Tibet air shower experiment has been observing the shadowing of galactic cosmic rays continuously by the moon and sun since 1990 [17]. We began construction of the Tibet-III air shower array in 1999 by increasing the number of detectors; we completed it in Fall of 2003. The present Tibet-III air shower array consists of 789 scintillation counters of $0.5 \mathrm{~m}^{2}$, each of which is placed in a $7.5 \mathrm{~m}$ square grid, covering a total area of about $37000 \mathrm{~m}^{2}$ [18].

In this paper, we present the result on the search for antiprotons based on the moon shadow observed during the period from 1999 through 2004 using the Tibet-III air shower array.

\section{Experiment}

The Tibet-III air shower array used in this experiment was constructed in 1999 at Yangbajing (4300 $\mathrm{m}$ a.s.l.) in Tibet and operated until 2004. The array, corresponding to the inner part of the full-scale Tibet-III air shower array, consists of 533 scintillation counters covering $22050 \mathrm{~m}^{2}$ [19,20]. The mode energy of detected events is about $3 \mathrm{TeV}$ for proton-induced showers and the angular resolution is $0.9^{\circ}$. The systematic error of the energy determination of primary particles and systematic pointing error of the array have been well calibrated by comparing the observed displacement of the moon shadow because of the geomagnetic field with the Monte Carlo simulation, as discussed in the paper [21].

With the advent of this array, we observed $6.1 \times 10^{10}$ events during the period from November 1999 through December 2004 (1041 live days in total). These events were selected by imposing the following conditions: 1) Each shower must fire four or more counters recording 1.25 or more particles; 2) all of fired counters or eight of nine fired counter which recorded the highest particle density must be inside the fiducial area; and 3) the zenith angle of the arrival direction must be less than $40^{\circ}$. After the these selections, $1.5 \times 10^{10}$ events remained for further analyses. 


\section{Simulation}

We have done a detailed Monte Carlo simulation to estimate the flux of antiprotons from the shape of the moon shadow by cosmic rays under the influence of geomagnetic field. A deviation of the observed shadow from the simulated one based on the normal primary cosmic rays (without antiparticles) would give the proof of the existence of antiprotons pouring on the Earth. For the normal primary cosmic rays, we used a best-fit curve for each component based on the experimental data measured by direct observations on board the balloon and satellite (for experimental data, see the paper by Gaisser et al. [22] and references therein).

We used the CORSIKA Ver. 6.200 code [23] with the QGSJET interaction model for generation of air showers in the atmosphere. All secondary particles produced by a primary particle were traced until their energies becomes 1 $\mathrm{MeV}$ in the atmosphere. Simulated air-shower events were then input to the detector with the same detector configuration as the Tibet-III air shower array using the Epics uv8.00 code [24] to calculate the energy deposit of these shower particles and were stored in the disk with the same format as the experimental data.

For the geomagnetic field, we used the Virtual Dipole Moment (VDM) model [25] in which the dipole moment at altitude $>600 \mathrm{~km}$ is taken to be $8.07 \times$ $10^{25} \mathrm{G} \cdot \mathrm{cm}^{3}$. We also made the simulation based on the International Geomagnetic Reference Field (IGRF) model [26] to compare with that based on the VDM model. The difference of the deflection of particle trajectries between the two models is then estimated to be within $5 \sim 7 \%$ in the part of the sky where the moon is visible by the Tibet-III air shower array. We found, however, that the IGRF model gives almost the same result as the VDM model for the actual moon shadow simulation in which the angular resolution of the array is taken into account. Hence, we adopted the results obtained based on the VDM model in this paper.

The simulation for the moon shadow was performed through the following steps:

(1) Primary particles in the energy range from $0.3 \mathrm{TeV}$ to $1000 \mathrm{TeV}$ are thrown isotropically toward the observation site on the top of the atmosphere along the revolution orbit of the moon. Air shower events generated by these particles in the atmosphere are then collected within the circle of $300 \mathrm{~m}$ radius from the center of an air shower array. This array has the same structure and detector response as the Tibet-III air shower array.

(2) All air shower events triggered by the air shower array are analyzed in the 
same way as the experimental data to estimate the size and the arrival direction of each event.

(3) An opposite charge is then assigned to the primary particle of each analyzed event; and these particles (anti-particles) are again shot back uniformly in the $\pm 10^{\circ} \times \pm 10^{\circ}$ direction centering on the moon: they originated from the air shower array at Yangbajing and tracked through the geomagnetic field up to the moon.

(4) We select only the events hitting the moon; these selected events are again traced back from the moon to the Earth after reversing the charge of each primary particle. The final direction of each cosmic ray is determined by smearing the difference between the moon direction and the estimated air shower direction where the angular resolution of the array is taken into account.

Using this method, we can simulate the moon shadow that is capable of direct comparison with the observed data.

\section{Data Analysis}

For analysis of the moon shadow, an on-source event is defined as an event which falls on the $\pm 5^{\circ} \times \pm 5^{\circ}$ square window centered at the moon direction. The background is estimated using the events coming from the following eight off-source windows of the same size as on-source. These positions are located on the same zenith angle as the moon, but separated by $\pm 5^{\circ}, \pm 10^{\circ}, \pm 15^{\circ}$, and $\pm 20^{\circ}$ in the azimuth angle. The number of events averaged over these eight off-source windows was taken to be the background [17].

Shown in Fig. 1 is the moon shadow observed using the events obtained for 1041 live days with the Tibet-III air shower array. The significance at the peak of the shadow is estimated as about $40 \sigma$ level and the shadow is shifted westward by about $0.23^{\circ}$. The mode energy of observed events is estimated as 3 $\mathrm{TeV}$ for proton-induced showers. Note that a $1 \mathrm{TeV}$ proton is shifted westward by $1.6^{\circ}$ at Yangbajing. This means that a major effect of antiprotons should appear in the eastward angular distance smaller than about $0.5^{\circ}$ on the moon shadow observed with the Tibet-III air shower array. The systematic error of the energy estimation of primary particle is estimated to be $\pm 8 \%$ level and the systematic pointing error of the array is smaller than $0.011^{\circ}$ [21]. Using these basic data and simulation results, we discuss the flux of antiprotons in the next section. 


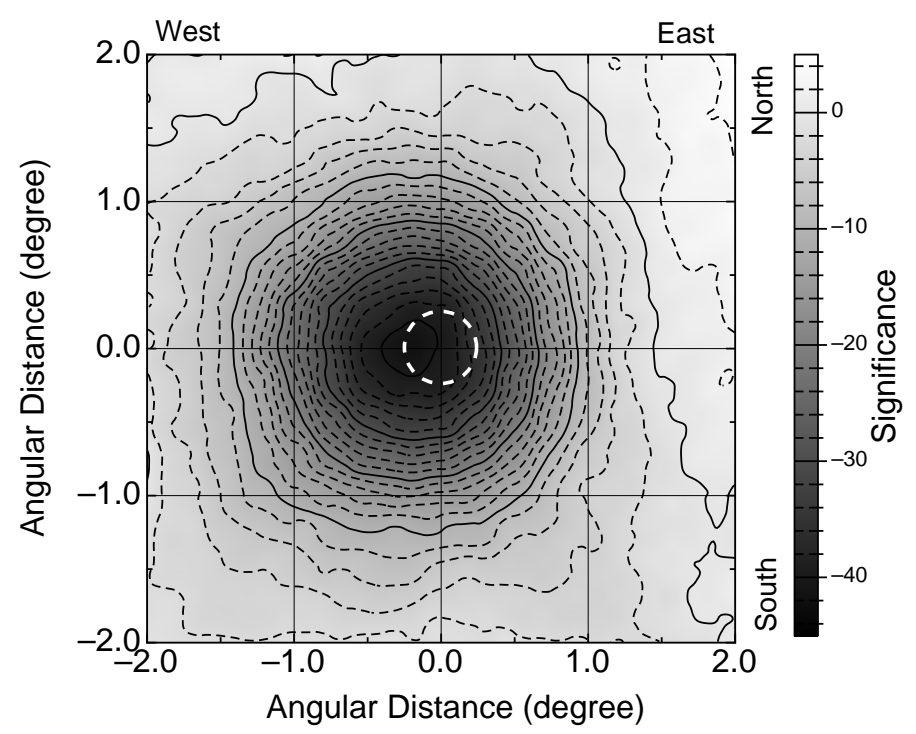

Fig. 1. Significance map of the deficit event densities observed with the Tibet-III air shower array for 1041 live days. The origin of the coordinate is taken in the direction of the moon. The white dashed circle denotes the apparent size and position of the moon. A right-hand side scale expresses the level of significance of the deficit event density in terms of the standard deviation $\sigma$. The significance at the central peak of the shadow is about $40 \sigma$ on the two-dimensional analysis [17].

\section{Results and Discussion}

Figure 2 shows the one-dimensional distribution of the observed deficit events around the moon in the north-south direction, where the vertical axis represents the number of deficit events inside the range of $\pm 1^{\circ}$ in the east-west direction. The origin of the coordinate is taken to indicate the apparent direction of the moon. The position of the shadow center is almost consistent with that of the moon because the east-west component of the geomagnetic field is negligibly small at Yangbajing latitude, so that the cosmic rays are not deflected in the north-south direction. As this figure shows, our data show good agreement with the MC simulation $\left(\chi^{2} /\right.$ d.o.f. $\left.\sim 1.15\right)$. The peak position of the distribution in the north-south direction is estimated as $0.0048^{\circ} \pm 0.011^{\circ}$.

Figure 3 shows the one-dimensional distribution of the observed deficit events around the moon in the east-west direction, where the vertical axis represents the number of deficit events inside the range of $\pm 1^{\circ}$ in the north-south direction. The observed moon shadow is shifted westward by $0.23^{\circ}$, which is consistent with the MC simulation. There exists, however, no evidence indicating deficits of cosmic rays at the opposite position around $\theta=0.23^{\circ}$ in the eastward direction, corresponding to the particles with negative charge (anti-matter) such as $\bar{p}, \overline{H e}, \bar{C}, .$. , and $\overline{F e}$, if any.

Next, to evaluate the contribution of antiprotons to the moon shadow, we 


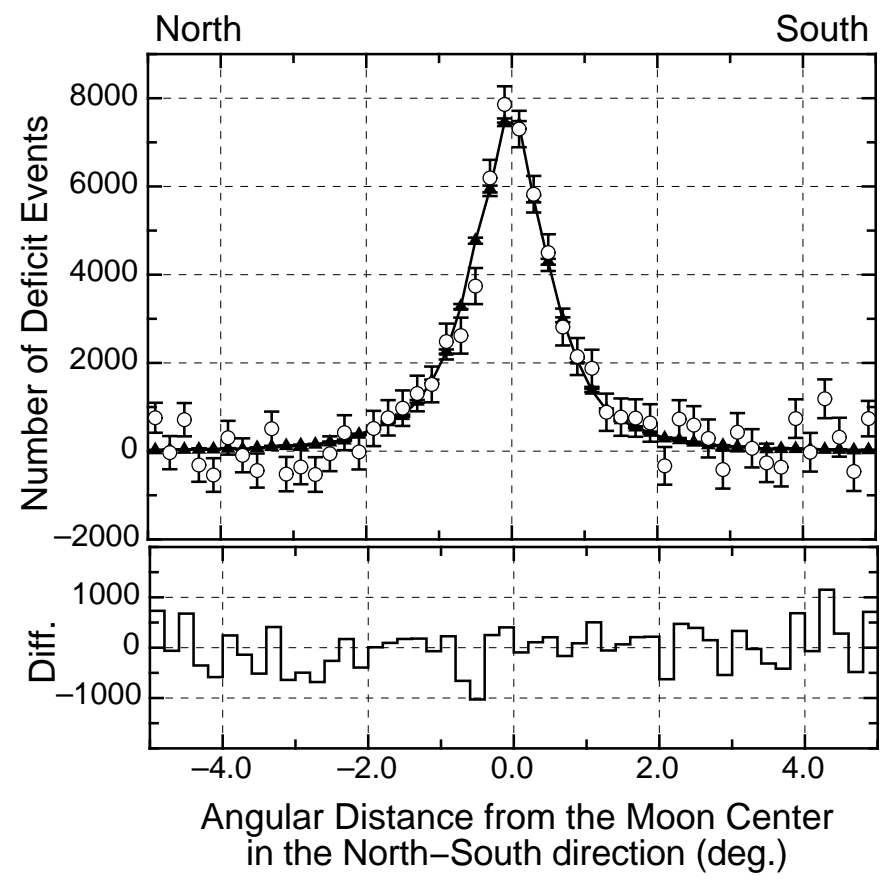

Fig. 2. One-dimensional distribution of the observed deficit events around the center of the moon in the north-south direction. The vertical axis represents the number of deficit events inside the range of $\pm 1^{\circ}$ in the east-west direction. The open circles denote the experimental data and the filled triangles denote the $\mathrm{MC}$ data. The solid line represents a best fit curve according to the function $f_{1}(\theta)$. Each error bar denotes $\pm 1 \sigma$. The histogram of the following figure shows the residual of the observed deficit event distribution to the function $f_{1}(\theta)$.

obtained two kinds of moon shadow that were cast by all cosmic rays and protons, respectively. We assume that the deficit event distribution in each shadow can be expressed as a superposition of Gaussian functions. It is found that four Gaussian functions are adequate for fitting to both distributions in the angular distance smaller than about $5^{\circ}$ from the center of the moon.

The moon shadow by all cosmic rays is then expressed as

$$
f_{1}(\theta)=\sum_{i=1}^{4} A_{a l l, i} \exp \left(-\frac{4 \ln 2 \times\left(\theta-M_{a l l, i}\right)^{2}}{\sigma_{a l l, i}^{2}}\right)
$$

The moon shadow by protons is also expressed as

$$
f_{2}(\theta)=\sum_{i=1}^{4} A_{p, i} \exp \left(-\frac{4 \ln 2 \times\left(\theta-M_{p, i}\right)^{2}}{\sigma_{p, i}^{2}}\right),
$$

where $\theta$ is the angular distance from the moon center in the west-east direction and $A, M$ and $\sigma$ are the fitting parameters for the distribution function, 


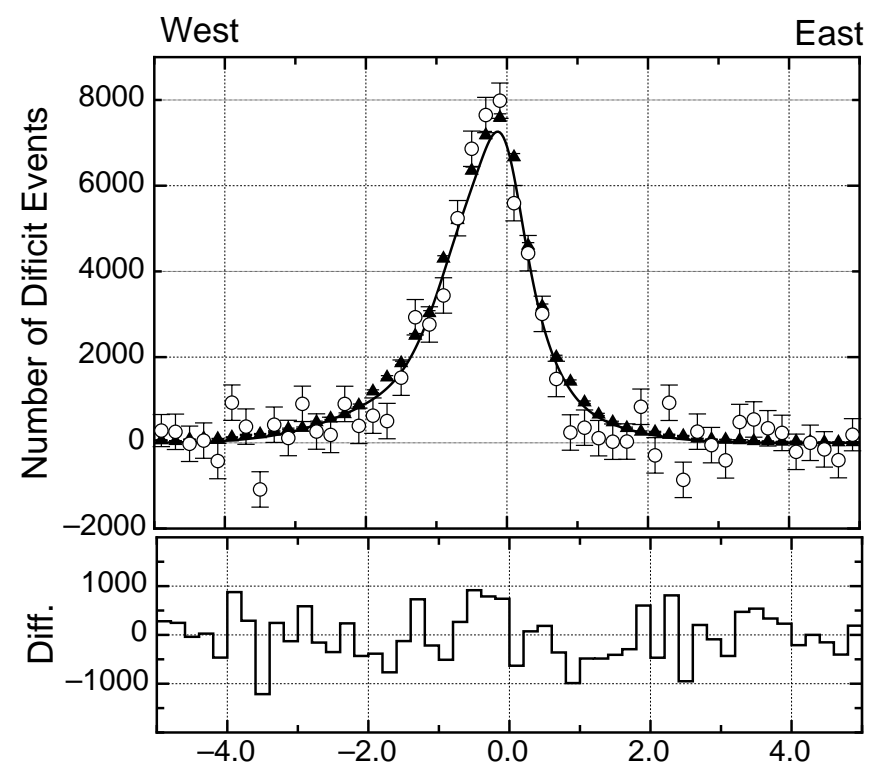

Angular Distance from the Moon Center in the East-West direction (deg.)

Fig. 3. One-dimensional distribution of the observed deficit events around the center of the moon in the east-west direction. The vertical axis represents the number of deficit events inside the range of $\pm 1^{\circ}$ in the north-south direction. The open circles denote the experimental data and the filled triangles denote the MC data without antiprotons. The solid line is a best-fit curve according to the function $f_{3}(\theta)$. Each error bar denotes $\pm 1 \sigma$. The histogram of the following figure shows the residual of the observed deficit event distribution to the function $f_{3}(\theta)$.

respectively, for all cosmic rays and protons.

On the other hand, the observed moon shadow should be expressed by the following function $f_{3}(\theta)$

$$
f_{3}(\theta)=a f_{1}(\theta)+b f_{2}(-\theta)
$$

where the first term represents the deficit in cosmic rays and the second term represents the deficit in antiprotons. From our simulation, the fraction of protons to all cosmic rays is estimated as $62 \pm 1 \%$ of all cosmic rays, so the ratio $b / 0.62 a$ corresponds to the $\bar{p} / p$ ratio. As shown in Fig. 3, the observed deficit events can be fitted by the function $f_{3}(\theta)$ with the parameters of $a=1.64 \pm 0.10$ and $b=-0.10 \pm 0.08\left(\chi^{2} /\right.$ d.o.f. $\left.\sim 1.50\right)$, where the fitting was made under the boundary condition that the integral of $a f_{1}(\theta)$ should be smaller than the total number of observed events.

To calculate an upper limit of the fraction of antiprotons, the confidence interval of the parameter $b$ is estimated using Feldman and Cousins Statistics [27] because parameter $b$ is negative. The upper limit of the $\bar{p} / p$ ratio at the $90 \%$ confidence level was then obtained as 0.07 . The present result is plotted 


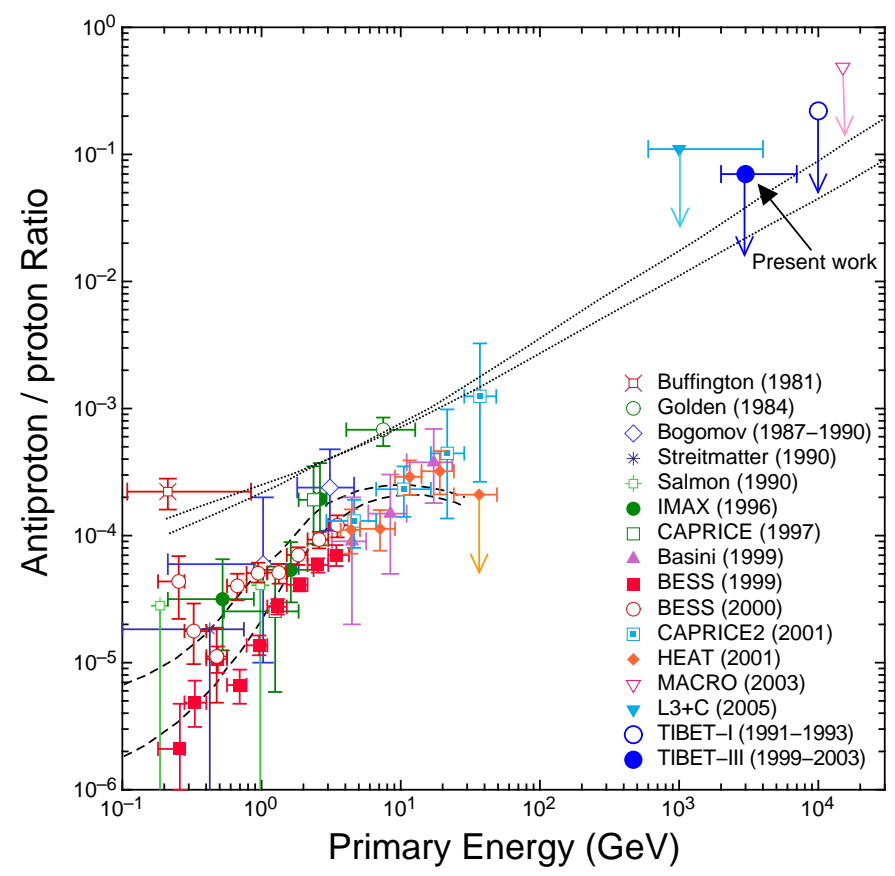

Fig. 4. The antiproton/proton ratio at the top of the atmosphere. The experimental results are as follows: Buffington (1981) [28], Golden (1984) [29], Bogomolov (1987-1990) [30], Salomon (1990) [31], IMAX [32], CAPRICE (1997) [33], Basini (1999) [1], BESS (1999) and BESS (2000) [4], CAPRICE2 (2001) [3], HEAT (2001) [2], L3+C (2005) [34], Streitmatter (1990) [35], MACRO (2003) [36], L3+C (2005) [34], and opened circles are those by TIBET-I (1990-1993) [16]. The present result by TIBET-III (1999-2004) is denoted by the filled circle. The dashed lines, upper and lower limit, are the calculation based on the leaky box model by Simon, Molnar $\&$ Roesler [6]. The dotted lines are for a model that includes both leaky box and the presence of antistars. The rigidity dependent confinement of cosmic rays in the Galaxy is assumed to be $\propto R^{-\delta}$, and two curves $A$ and $B$ are the cases of $\delta=0.7$ and 0.6 , respectively [12].

in Fig. 4, together with other results.

In this figure, four experimental data are plotted at multi-TeV energies. Among them, two are from the Tibet air-shower experiment using the moon shadow (present work) and the sun shadow (Tibet-I [16]). The present result gives the most stringent constraint on the $\bar{p} / p$ ratio compared to other data.

In conclusion, we showed that the moon shadow observation can provide unique data on the $\bar{p} / p$ ratio at the multi-TeV region. A further observation of the moon shadow and a fine tuning of the $\mathrm{MC}$ simulation will give a more stringent constraint to the flux ratio of antiprotons to protons in the very near future. If these data are combined with the new data that are obtainable in the energy region around $100 \mathrm{GeV}$ by the PAMELA satellite mission (launched on board the Russian satellite in 2006) [37] and by the forthcoming space experiment AMS [38], we might be able to get new information about 
the production of antiprotons in the TeV energy region.

\section{Acknowledgments}

The collaborative experiment of the Tibet Air Shower Arrays has been performed under the auspices of the Ministry of Science and Technology of China and the Ministry of Foreign Affairs of Japan. This work was supported in part by Grants-in-Aid for Scientific Research on Priority Areas (712) (MEXT) and by Scientific Research (JSPS) in Japan, and by the Committee of the Natural Science Foundation and by the Chinese Academy of Sciences in China.

\section{References}

[1] Basini, G. et al., Proc. 26th Int. Cosmic Ray Conf. (Salt Lake City) 3 (1999) 77.

[2] Beach, A.S. et al., Phys. Rev. Lett. 87 (2001) 271101.

[3] Boezio, M. et al., Astrophys. J. 561 (2001) 787.

[4] Asaoka, Y. et al., Phys. Rev. Lett. 88 (2002) 051101.

[5] Strong, A.W. et al, Astrophys. J, 613 (2004) 962 ; astro-ph/0701517 (2007)

[6] Simon, M., Molnar, A. \& Roesler, S., Astrophys. J. 499 (1998) 250.

[7] Meneguzzi, M., Audouze, J., Reeves, H., Astron. \& Astrophys. 15 (1971) 337.

[8] Gaisser, T.K., Cosmic Rays and Particle Physics (Cambridge Univ. Press), 1990.

[9] Engelmann, J.J. et al., Astron. \& Astrophys. 233 (1990) 96.

[10] Peters, B. \& Westergård, N.J., Astrophys. Sp. Sci. 48 (1977) 21.

[11] Protheroe, R.J., Astrophys. J. 251 (1981) 387.

[12] Stephen, S.A. \& Golden, R.L., Space Sci. Rev. 46 (1987) 31.

[13] Barrau, A. et al., Phys. Rev. D 72 (2005) 063507.

[14] Urban, M. et al., Nucl. Phys. B (Proc. Suppl.) 14B (1990) 223.

[15] Amenomori, M. et al., Phys. Rev. Lett. 69 (1992) 2468.

[16] Amenomori, M. et al., Proc. 24th Int. Cosmic Ray Conf. (Roma) 3 (1995) 84.

[17] Amenomori, M. et al., Phys. Rev. D 47 (1993) 2675. 
[18] Amenomori, M., et al., Proc. of 27th Int. Cosmic Ray Conf. (Tsukuba) 5 (2003) 3019 .

[19] Amenomori, M. et al., Astrophys. J. 598 (2003) 242.

[20] Amenomori, M. et al., Science 314 (2006) 439.

[21] Amenomori, M. et al., Proc 29th Int. Cosmic Ray Conf. (Pune) 6 (2005) 53.

[22] Gaisser, T.K. et al., Proc. 26th Int. Cosmic Ray Conf. (Hamburg) 5 (2001) 1643.

[23] Heck, D. et al., Report FZKA, 6019, Forschungszentrum Karlsruhe, 1998.

[24] Kasahara, K., http://cosmos.n.kanagawa-u.ac.jp/EPICSHome.

[25] For example, S. Chapman and J. Bartels, Geomagnetism (Oxford University Press), London, 1940.

[26] For example, http://www.ngdc.noaa.gov/IAGA/vmod/igrf.html.

[27] Feldman, G.J., and Cousins, R.D., Phys. Rev. D 57 (1998) 3873.

[28] Buffington, A., Schindler, S. M. \& Pennypacker, C. R., Astrophys. J. 248 (1981) 1179 .

[29] Golden, R. L. et al., Astrophys. Lett. 24 (1984) 75.

[30] Bogomolov, E. A. et al., Proc. 20th Int. Cosmic Ray Conf. (Moscow) 2 (1987) 72 .

[31] Salomon, M. H. et al., Astrophys. J. 349 (1990) 78.

[32] Mitchell, J.W. et al., Phys. Rev. Lett. 87 (1996) 3057.

[33] Boezio, M. et al., Astrophys. J. 487 (1997) 415.

[34] Achard, P. et al., Astropart. Phys. 23 (2005) 411.

[35] Streitmatter, R. E. et al., Proc. 21st Int. Cosmic Ray Conf. (Adelaide) 3 (1990) 277.

[36] Ambrosio, M. et al., Astropart. Phys. 20 (2003) 145.

[37] Picozza, P. et al. Astropart. Phys. 27 (2007) 296 ; http://wizard.roma2.infn.it/pamela/.

[38] AMS Collaboration, http://ams.cern.ch/. 